PART III

REFLECTION NEBULAE AND

DIFFUSE GALACTIC LIGHT 


\title{
SURFACE POLARIMETRY OF THE MILKY WAY
}

\author{
RAMON D. WOLSTENCROFT \\ Institute for Astronomy, University of Hawaii, H.I., U.S.A.
}

\begin{abstract}
Two methods of determining the state of polarization of the integrated light from the Milky Way are discussed. In the symmetry method it is assumed that the polarized foreground zodiacal light possesses north/south symmetry relative to the ecliptic: an average polarized intensity of at least $10 S_{10}(R)$ is derived for the Milky Way at $l \simeq 90^{\circ}$ and $270^{\circ}$, which is consistent with the result of Sparrow and Ney (1972) who obtained $18 S_{10}(R)$ at $l=35^{\circ}$ and $210^{\circ}$. In the method of two epochs the above assumption is not needed but instead time variations of the zodiacal light are assumed to be insignificant: the results obtained by this method (in $S_{10}(V)$ units), although compatible with the values found by the symmetry method, do however show that time variations may have influenced this result. Although the evidence for a definite polarization of the Milky Way is persuasive it cannot yet be considered conclusive.
\end{abstract}

\section{Introduction}

The integrated light from the Milky Way is the sum of integrated starlight and diffuse galactic light, both of which are expected to be polarized. If the polarization is large enough to be measured reliably, surface polarimetry of the Milky Way could very probably provide valuable new information on the properties of the interstellar grains. In this paper we confine our attention to the question: 'is the Milky Way appreciably polarized?'

To determine the polarization of the Milky Way from observations of the night sky, corrections must be made to allow for the strongly polarized foreground zodiacal light and also care must be exercized in observing only at those optical depths where the influence of tropospheric scattering may be neglected. In order to make an intelligent estimate of the foreground zodiacal light it is necessary to know how the zodiacal light polarization varies with time and angular coordinates. Unfortunately our knowledge of this is quite poor and we are forced to make assumptions which although plausible may conceivably be greatly in error. Two such assumptions are: (1) the time variations of the zodiacal light are insignificant; and (2) the zodiacal light at a given instant has the same properties at mirror points in solar ecliptic coordinates, i.e., at $\left(\beta, \lambda-\lambda_{\odot}\right),\left(-\beta, \lambda-\lambda_{\odot}\right),\left(\beta, \lambda_{\odot}-\lambda\right)$ and $\left(-\beta, \lambda_{\odot}-\lambda\right)$, where $\left|\lambda-\lambda_{\odot}\right|$ runs from $0^{\circ}$ to $180^{\circ}$. Schmidt and Leinert (1966), using both assumptions, obtained a degree of polarization, $p$, in the range $0,3 \%$ to $2.3 \%$ at six longitudes between $l=26^{\circ}$ and $122^{\circ}$ $\left(|b|<10^{\circ}\right)$. Sparrow and Ney (1972), using assumption (1) only, determined values of the polarized intensity at $l=35^{\circ}$ and $210^{\circ}$ of $4 S_{10}$ (blue) and $18 S_{10}$ (red): the former value corresponds to $p \simeq 2 \%$. The observations of Schmidt and Leinert had to be corrected for scattered artificial light and thus should be considered less reliable than those of Sparrow and Ney which were obtained from the OSO-5 satellite. 


\section{The Symmetry Method}

In an ideal approach to this problem the distribution of polarization over the entire sky should be determined at monthly intervals throughout the year: this would permit the assumptions (1) and (2) to be checked and if valid the polarization of the Milky Way could be deduced at all longitudes. In a more practical approach we have determined the distribution of polarization over the sky at a single epoch and have applied assumption (2); we shall call this the symmetry method. The measurements we have used were obtained during a three-week period at Chacaltaya, Bolivia, in
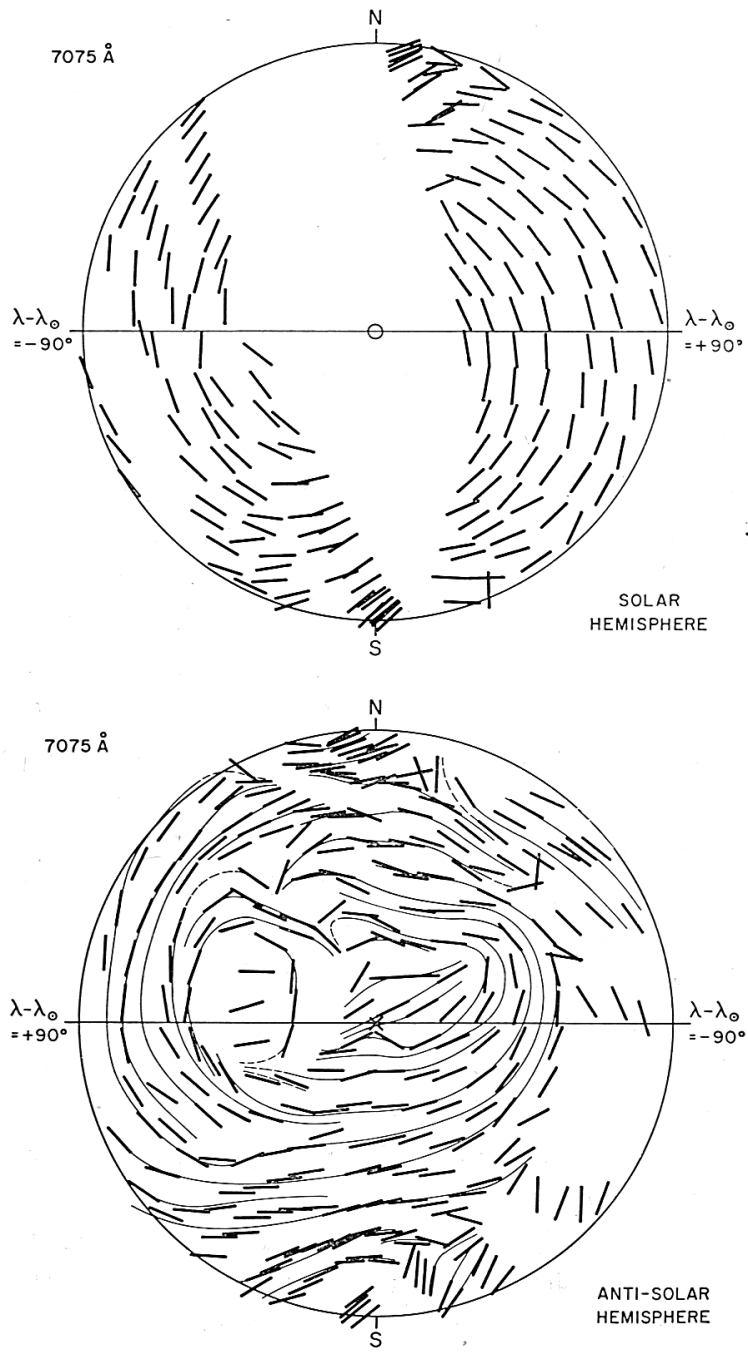

Fig. 1. Orientation of the polarization plane of the night sky at $7075 \AA$ during August, 1964. In the anti-solar hemisphere streamlines are drawn parallel to the polarization vectors. The Sun and anti-solar point are indicated by an open circle and a cross respectively. 
August 1964. The equipment has been described elsewhere (Wolstencroft and Brandt, 1967). The orientation of the polarization plane of the observed night sky radiation at $7075 \AA$ is shown in Figure 1. In the solar hemisphere the polarization orientations define a circular pattern centered on the Sun supporting the widely held view that the zodiacal light is the principal source of the polarization in most of this hemisphere. In the anti-solar hemisphere this circular pattern is seen in the outer parts of the distribution, although it is somewhat distorted: closer to the anti-solar point the distribution is markedly complex and the origin of the polarization is less certain. The average polarized intensity, $p I$, at $7075 \AA$ is given in Figure 2 and Table I for $10^{\circ} \times 10^{\circ}$ areas in $\left(\beta, \lambda-\lambda_{\odot}\right)$ for $\left|\lambda-\lambda_{\odot}\right|>60^{\circ}$. A minimum of 50 and an average of 200 observations per area were used: this is also true for Figure 1. The mean $\left(\beta, \lambda-\lambda_{\odot}\right)$ for each $10^{\circ} \times 10^{\circ}$ area is not in general at the center of the area and no attempt has been made to correct for this. Although these measurements were made at a high altitude site $(h=5.2 \mathrm{~km})$ in the red $(\tau=0.08$ at the zenith) the influence of tropospheric scattering cannot be ignored. The dependence of $p I$ on air mass has been examined for each $10^{\circ} \times 10^{\circ}$ area: in most cases $p I$ is independent of air mass for $z<70^{\circ}$ to within $\pm 2 S_{10}(R)$ for regions with $p I<20 S_{10}(R)$ (the unit used throughout this paper is $S_{10}(R, \mathrm{~A} 0 \mathrm{~V})$, abbreviated to $S_{10}(R)$; it is the equivalent number of A0V stars of magnitude $R=10$ per sq deg). The values of $p I$ in Figure 2 and Table $I$ are free of the influence of tropospheric scattering to these limits. The influence of tropospheric scattering on the polarization orientation is more severe, particularly for regions of

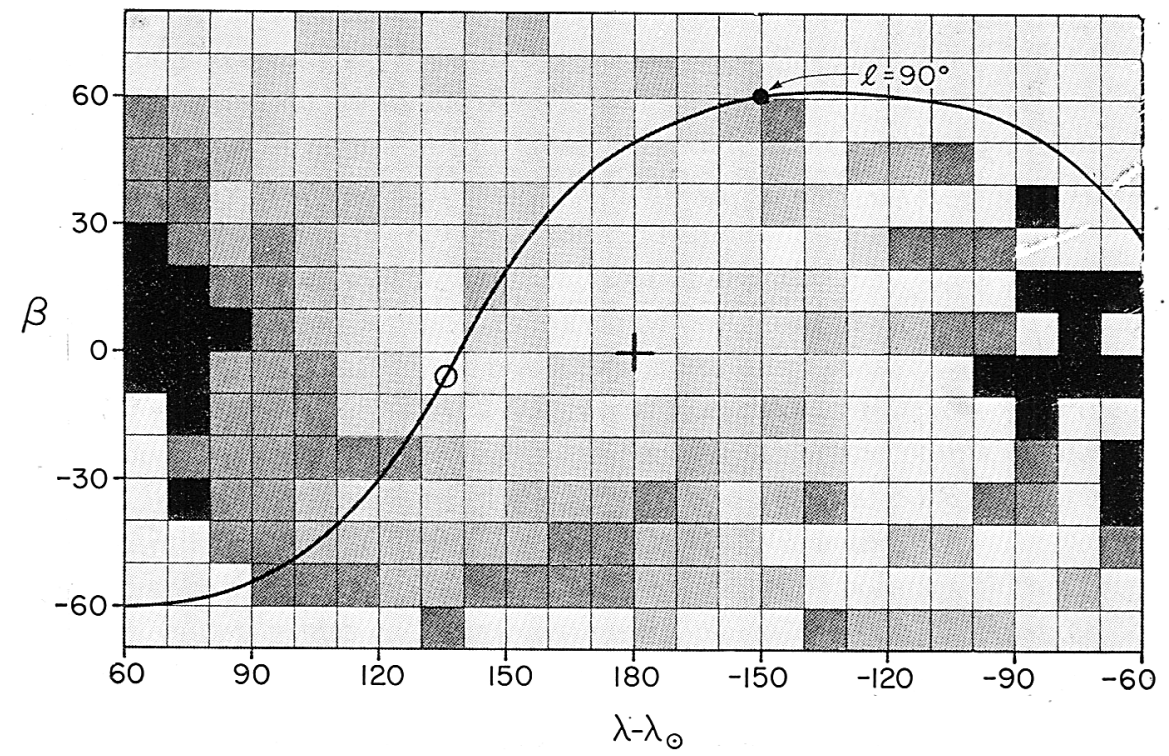

Fig. 2. The average polarized intensity in $10^{\circ} \times 10^{\circ}$ areas of the night sky at $7075 \AA$ in $S_{10}(R, A 0 \mathrm{~V})$ units during August 1964, The polarized intensity increases as the shading goes from lightest to darkest in the sequence the sequence 1 to 7,8 to 14,15 to 21,22 to 43 and 44 and greater in $S_{10}(\mathrm{R}, \mathrm{A} 0 \mathrm{~V})$ units. There is no data for areas without shading. The continuous line inclined to the ecliptic is the galactic equator; the open circle represents the galactic center. 


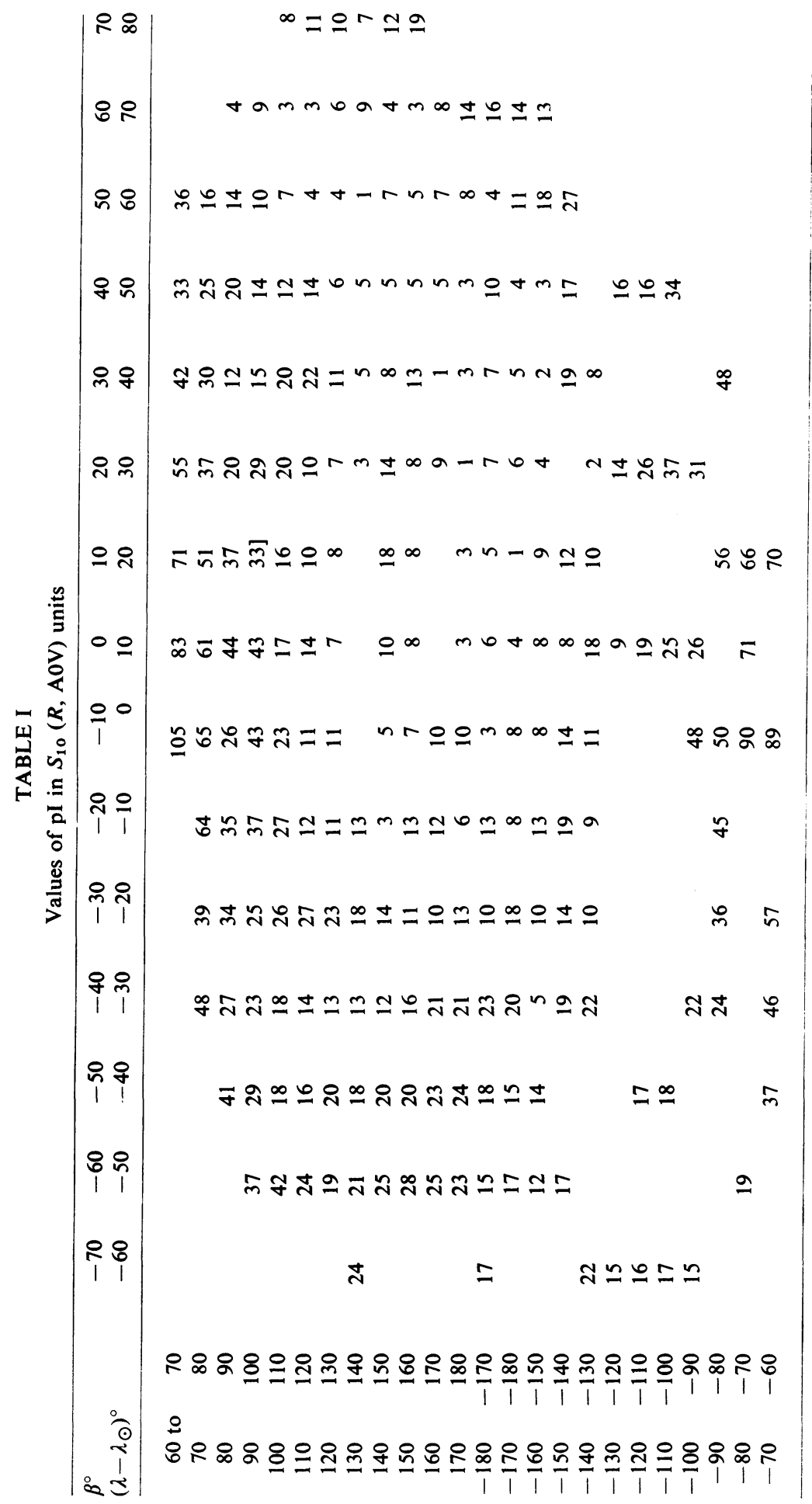


small $p I$; no selection of data at small air mass was made in preparing Figure 1 so that some of the true structure is distorted, for example that near the ecliptic poles.

The distribution of polarized intensities shows several unexpected features. The smallest values of $p I$ are not centered on the anti-solar point, as would be expected, but instead define a region mostly in the northern ecliptic hemisphere which is inclined to the ecliptic by about $40^{\circ}$ with a centroid at about $\beta=30^{\circ}, \lambda-\lambda_{\odot}=160^{\circ}$. The galactic plane passes close to the centroid, although because of the great extent of the region of low $p I$ this should probably be considered a coincidence. In the evening hemisphere the values of $p I$ are considerably greater in the south than at mirror points in the north and this may also be true in the morning hemisphere although there is less data there. It is also interesting to note that the polarization orientations in the anti-solar hemisphere are rather more ordered in regions well away from the galactic plane. The possible interpretations of the asymmetry in $p I$ are: (a) the zodiacal light is asymmetric relative to the ecliptic and the anti-solar meridian and the polarization of the Milky Way is negligible; (b) the zodiacal light is symmetric and the Milky Way is polarized; (c) a combination of (a) and (b). We cannot yet distinguish with certainty between possibilities (a) and (b). However, it is worth noting that current analysis of data at $z=70^{\circ}$ to $80^{\circ}$ (not included in Figure 2) for which a moderate correction for tropospheric scattering is necessary, indicates that the values of $p I$ in the south/morning quadrant are less than in the south/evening quadrant but greater than in the north/evening quadrant: in order to draw conclusions about the Milky Way we shall adopt assumption (b).

In Figure $3 p I$ is plotted against $|\beta|$ for constant values of $\lambda-\lambda_{\odot}$. The galactic latitude is indicated only for low latitude points $\left(b<30^{\circ}\right)$. The error bar is the standard deviation based on about 200 observations for each $10^{\circ} \times 10^{\circ}$ area. The difference between the curves at high and low galactic latitude represents a lower limit to the polarized intensity of the galactic component since $p I$ is a vector quantity. The observations by Sparrow and Ney (1972) at high galactic latitude are shown for $\lambda-\lambda_{\odot}=$ $90^{\circ} / 100^{\circ}$; they agree very well with our values although this may be fortuitous because it is not certain that their unit, $S_{10}$ (red), coincides with our $S_{10}(R, \mathrm{~A} 0 \mathrm{~V})$ unit. The high galactic latitude points in the north and south differ on average by $4 S_{10}(R)$ for the six such points in Figure 3. At $\lambda-\lambda_{\odot}=80^{\circ}$ to $110^{\circ}$ and $160^{\circ}$ to $-150^{\circ}$ the points of low $b$ have greater and lesser values respectively than the points of high $b$; this would be true if the orientations of the galactic and zodiacal polarizations were respectively parallel or perpendicular (approximately) to one another.

The difference between the high and low galactic latitude points is plotted (Figure 4) for various values of the galactic longitude at which the line of constant $\left(\lambda-\lambda_{\odot}\right)$ crosses the galactic equator. A few negative values are present because ecliptic south minus north values, rather than the modulus of the difference were used. Average values for the the two ranges of $l$ at $55^{\circ}$ to $92^{\circ}$ and $278^{\circ}$ to $289^{\circ}$ are shown; we may use the curves to estimate an approximate value of $p l$ for the galactic component; the average value for the two ranges of $l$ is $9 S_{10}(R)$ which, since it is strictly a lower limit, is consistent with the value $18 S_{10}(R)$ obtained by Sparrow and Ney for $l=$ 


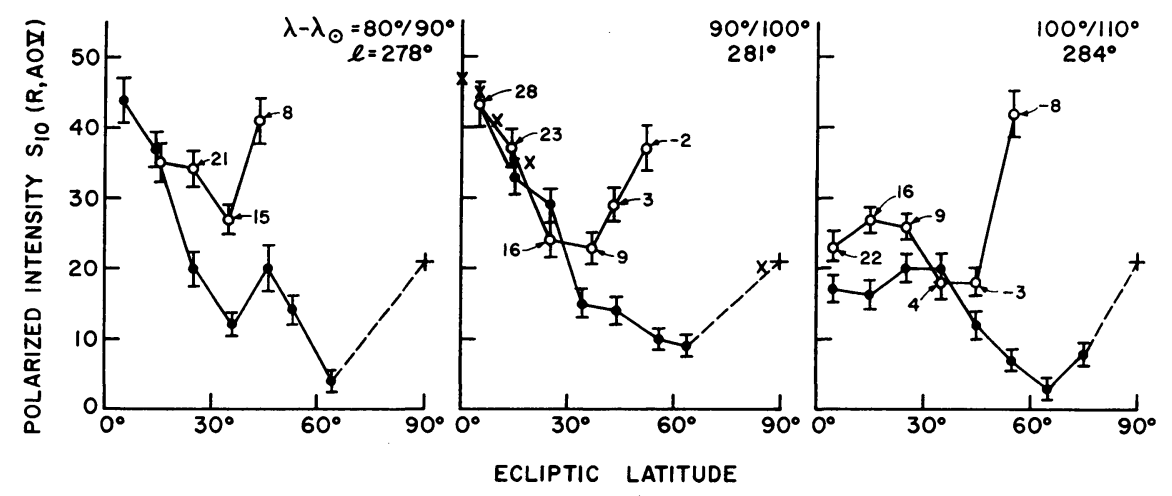

(Fig. 3a)

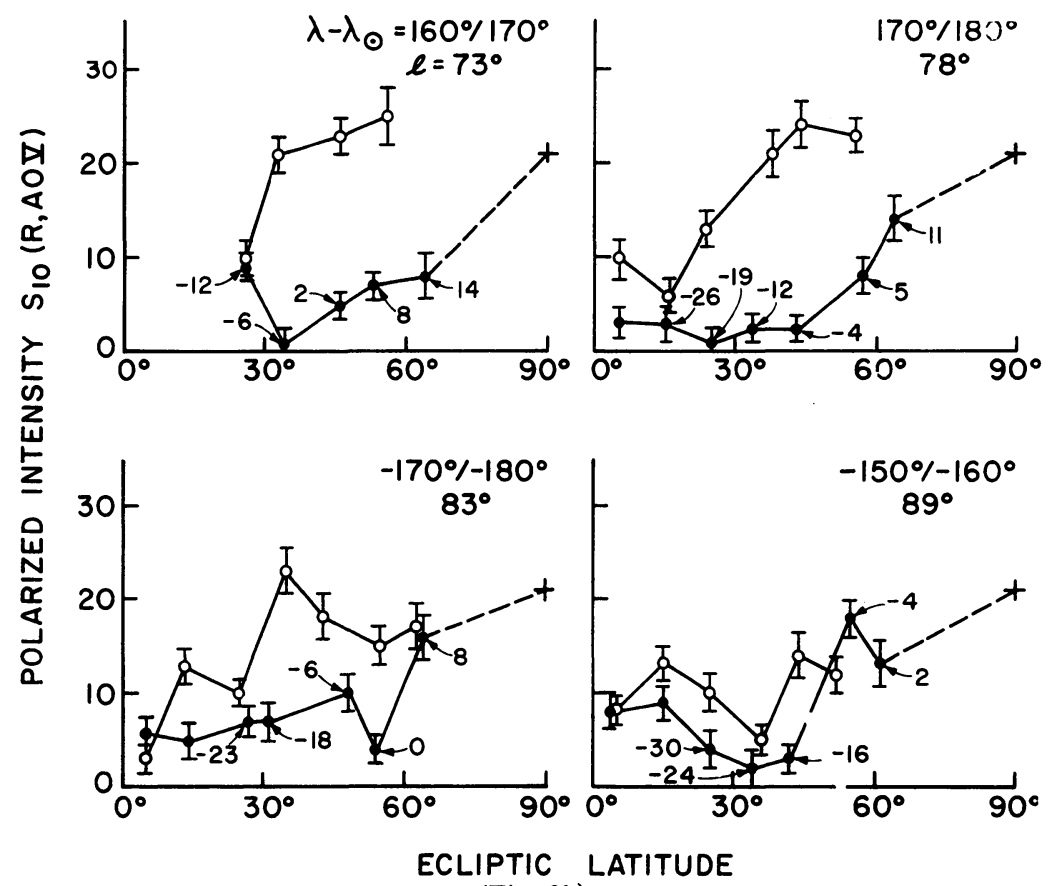

(Fig. 3b)

Fig. 3. Polarized intensity at a fixed $\lambda-\lambda_{\odot}$ vs $|\beta|$ derived from Table $I$ and Figure 2. Filled and open circles denote respectively points at northern and southern ecliptic latitudes. The number alongside certain points is the galactic latitude for points of low $b$; for points without numbers $b>30^{\circ}$. The cross at $|\beta|=90^{\circ}$ represents the average value for the northern ecliptic pole after applying an empirical correction for tropospheric scattering (the error of this point is estimated to be \pm 7$)$. At $\lambda-\lambda_{\odot}=90^{\circ} / 100^{\circ} X$ denotes high galactic latitude observations by Sparrow and Ney (1972).

$35^{\circ}$ and $210^{\circ}$. A more precise estimate of the galactic polarization will be obtained by subtraction of the Stokes parameters $(Q, U)$ in opposite ecliptic hemispheres once the polarization orientations uninfluenced by trophospheric scattering have been derived; 

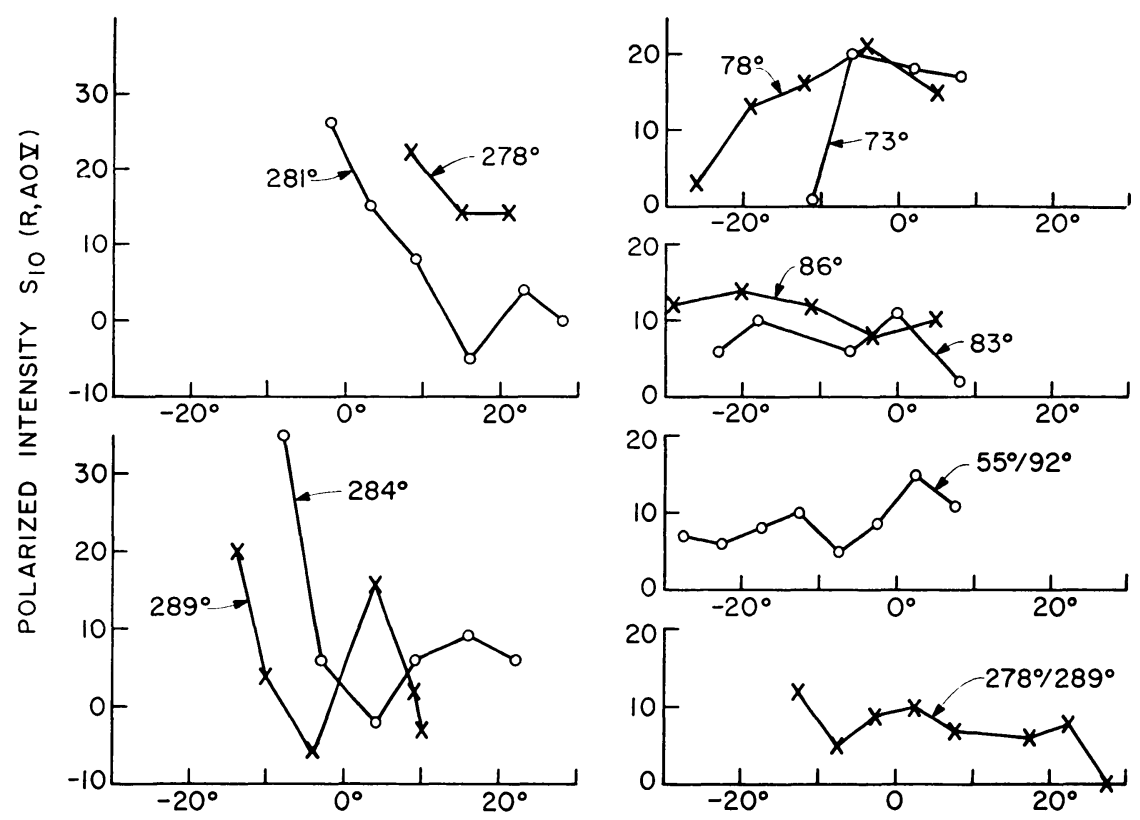

GALACTIC LATITUDE

Fig. 4. Difference of polarized intensity $\left(S_{10}(R, \mathrm{~A} 0 \mathrm{~V})\right.$ units) between high and low galactic latitude points of equal $1 \beta$ and $\lambda-\lambda_{\odot}$ vs the low galactic latitude coordinate. The number against each curve is the galactic longitude at which the great circle of constant $(\lambda-\lambda \odot)$ crosses the galactic equator.

The curves $55^{\circ} / 92^{\circ}$ and $278^{\circ} / 298^{\circ}$ represent averages over their respective longitude ranges.

the necessary assumption in carrying this out is that polarization orientations of the zodiacal light in the two ecliptic hemispheres are identical when the hemispheres are folded into one another about the ecliptic.

The method described above assumes that no significant time variations occurred during the three week observing period; in fact, all nights except one were contained in a one week period and further the observations were made close to the minimum of the solar cycle, so that this assumption is probably valid. The correctness of the assumption that the zodiacal light is symmetric in the four ecliptic quadrants is difficult to asses. It seems likely that the nodes and perihelia of the orbital planes of the dust particles are randomly distributed so that no east/west asymmetry is present; although the mean orbital plane of all particles is quite possibly inclined by a small angle to the ecliptic plane it is an open question whether this could produce a measurable north/south asymmetry.

\section{The Method of Two Epochs}

We have also carried out a study which makes no assumption about the angular distribution of the zodiacal light: the only assumption is that the time variations of the zodiacal light are insignificant. Observations were made of (a) a region in the Milky 
Way of low galactic latitude at an epoch $(1=1969$ January $17 / 18)$ when a region $\left(\beta, \lambda-\lambda_{\odot}\right)$ of zodiacal light of small polarized intensity was in the foreground and (b) the same region of zodiacal light $\left(\beta, \lambda-\lambda_{\odot}\right)$ at a later epoch $(2=1969$ May 19/20) when the background was a region of moderate or high galactic latitude. The observed (O) Stokes parameters of the night sky at epoch $j$ are related to the parameters for the Milky Way $(G)$, zodiacal light $(Z)$ and light scattered in the troposphere $(S)$ by

$$
\begin{aligned}
& Q_{o_{j}}=T_{j}\left(Q_{G j}+Q_{Z_{j}}\right)+Q_{S_{j}} \\
& U_{o_{j}}=T_{j}\left(U_{G j}+U_{Z_{j}}\right)+U_{S_{j}},
\end{aligned} \quad(j=1 \text { or } 2)
$$

where $T$ is the atmospheric transmission. By assuming that (i) the zodiacal component is the same at both epochs, (ii) the galactic component is negligible at $b>40^{\circ}$ and (iii) that tropospheric scattering is insignificant the galactic component of polarization may be deduced by subtraction, viz.

$$
\begin{aligned}
& Q_{G 1}=\frac{1}{T_{1}}\left[\left(Q_{o 1}-Q_{o 2}\right)+\left(T_{2}-T_{1}\right) Q_{Z}\right] \\
& U_{G 1}=\frac{1}{T_{1}}\left[\left(U_{O 1}-U_{o 2}\right)+\left(T_{2}-T_{1}\right) U_{Z}\right],
\end{aligned}
$$

where $Q_{\mathrm{Z}}=Q_{\mathrm{z}_{1}}=Q_{\mathrm{z}_{2}}$ and $U_{\mathrm{Z}}=U_{\mathrm{Z}_{1}}=U_{\mathrm{z}_{2}}$. The validity of the first two assumptions cannot be directly tested but this may be done for the third assumption. Observations were made at Haleakala, Hawaii at $\lambda=5080 \AA$ ( $\tau=0.12$ in the zenith) at air masses less than 1.15: we may place an upper limit on the polarized intensity of the light scattered in the troposphere of about $2 S_{10}(V)$ on the basis of both empirical studies carried out at Chacaltaya and theoretical studies (Wolstencroft and Van Breda, 1967).

The results are shown in Figures 5 and 6. Figure 5 shows the dependence of the polarized intensity $p_{G 1} I_{G 1}=\left(Q_{G 1}{ }^{2}+U_{G 1}\right)^{\frac{1}{2}}$ on $b$ for four $10^{\circ}$ ranges of longitude between $155^{\circ}$ and $195^{\circ}$. When $p_{G 1} I_{G 1}$ is averaged over all longitudes we obtain the values $6.6,8.8,11.4,9.8,7.7,6.9,6.1,3.8$ and $6.3 S_{10}(V)$ at $l=3^{\circ}$ to $19^{\circ}$ (in $2^{\circ}$ steps). The approximately linear trend for $b<7^{\circ}$ when extrapolated to $b=0^{\circ}$ gives $3 S_{10}(V)$. The orientation $\chi_{G 1}$ of the polarization plane vs $b$ (Figure 6) is measured from the direction of the north galactic pole in a counter clockwise direction as seen from outside the celestial sphere. The average standard error of a single point is $15^{\circ}$. For $l=160^{\circ}$ the average value of $\chi_{G 1}$ is close to $90^{\circ}$, i.e., the polarization plane is parallel to the galactic equator, but with increasing $l$ the average value increases to $120^{\circ}$ at $l$ $=190^{\circ}$. At each longitude $\chi_{G 1}$ shows a maximum at $b=11^{\circ} \pm 2^{\circ}$ : the maximum in $p_{G 1} I_{G 1}$ occurs at a different latitude, namely $b<8^{\circ}$.

While the values of $p I$ are approximately consistent with data in the red discussed earlier in this paper (see also Sparrow and Ney for a discussion of the color of $p I$ ) nevertheless, the assumption that the time variations of the zodiacal light may be ignored is a moot point. Fortunately the assumption may be tested. We have sought a correlation between the polarized intensities of the deduced galactic component 


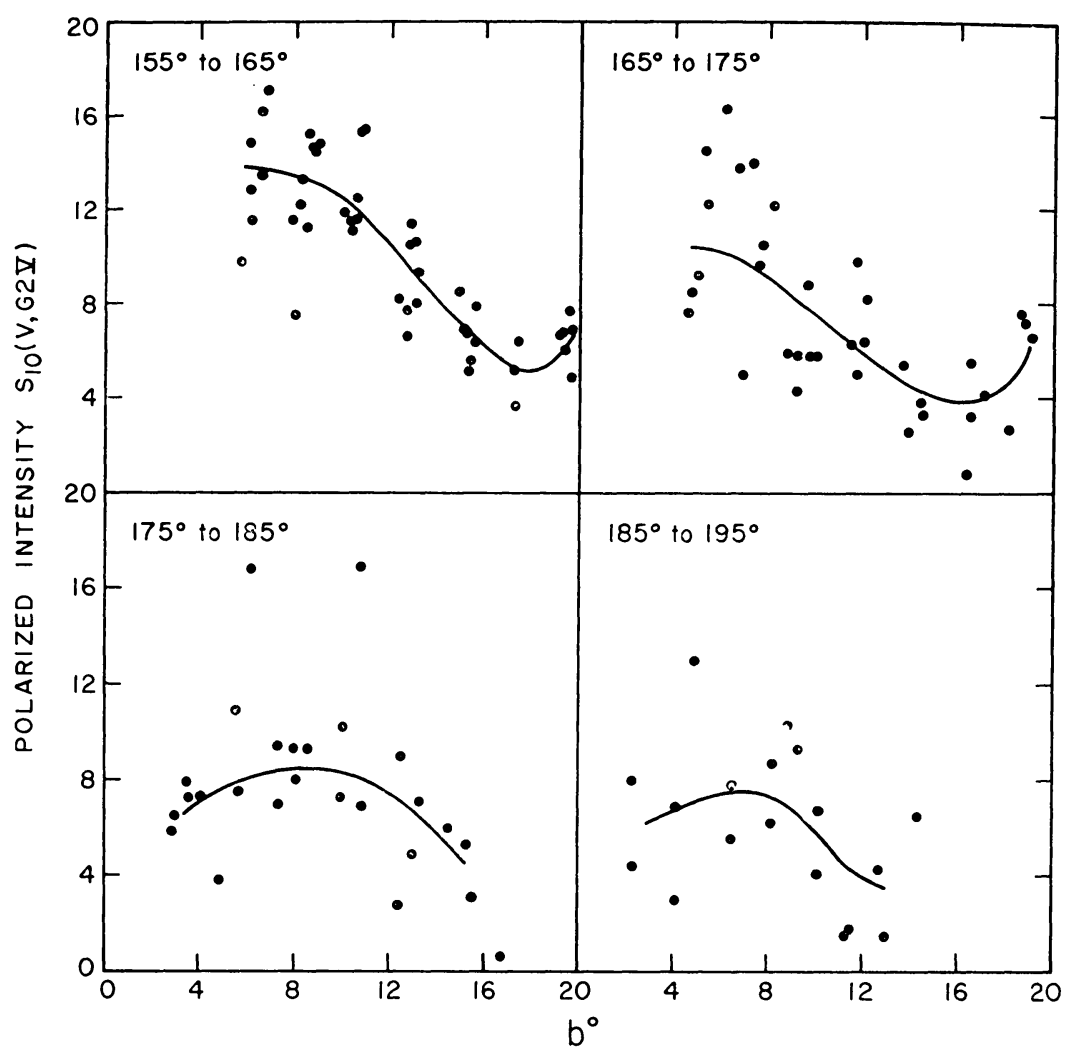

Fig. 5. Polarized intensity of the light from the Milky Way $\left(S_{10}(V, G 2 V)\right.$ units $)$ obtained by the method of two epochs for four longitude ranges from $155^{\circ}$ to $195^{\circ}$.

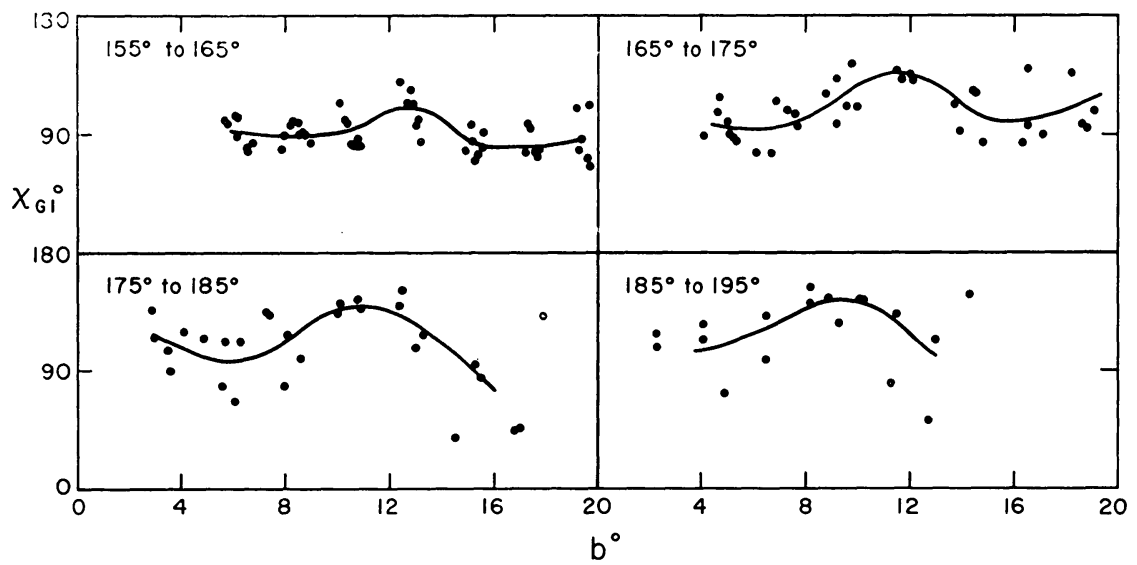

Fig. 6. Orientation of the polarization plane of the light from the Milky Way (method of two epochs): the orientation is measured counter-clockwise from the direction of the north galactic pole as seen from outside the celestial sphere. 
(measured at epoch 2). Unfortunately a moderate correlation exists which implies that the zodiacal component was not completely cancelled, that is, the zodiacal component was different at the two epochs. Further analysis is needed before the extent of the variation may be estimated and its influence on the deduced galactic polarization can be evaluated.

\section{Conclusions}

Of the two methods studied, reliable results have been obtained only by the symmetry method. Assuming that the zodiacal light possesses north/south symmetry with respect to the ecliptic we deduce that the polarized intensity of the Milky Way is at least $10 S_{10}(R)$ : this is consistent with the results of Sparrow and Ney (1972), who assumed time variations of the zodiacal light are insignificant. Before the reality of the galactic polarization can be established using this approach, the north/south asymmetry of the zodiacal light must be demonstrated.

\section{Acknowledgements}

This study was supported by NSF under Grant \#GA 28201 and by NASA under contract \#NAS 5 11303. The computations were performed by Jerry D. Wilson whose assistance is gratefully acknowledged. I thank Lothar W. Bandermann for helpful discussions.

\section{References}

Schmidt, T. and Leinert, C.: 1966, Z. Astrophys. 64, 110.

Sparrow, J. G. and Ney, E. P., 1972, Astrophys. J. 174, 705.

Wolstencroft, R. D. and Brandt, J. C.: 1967, in J. L. Weinberg (ed.), The Zodiacal Light and the Interplanetary Medium, NASA Sp-150, Washington, D.C., p. 57.

Wolstencroft, R. D. and van Breda, I. G.: 1967, Astrophys. J. 147, 255. 